(available at www.cbtinschizophrenia.com) are of interest and may have implications for clinical practice. In particular, the National Institute for Health and Care Excellence's ${ }^{2}$ (NICE) current recommendations that at least 16 sessions of therapy should be provided now seem open to question. As noted by Taylor \& Perera ${ }^{3}$ in their recent editorial in the BJPsych, this view is not evidence-based; instead it appears to derive from an impression gained by the Guideline Development Group that 'the evidence for CBT is primarily driven by studies that included at least 16 planned sessions' (NICE, ${ }^{2}$ p. 240).

Our database might additionally be used to examine two important claims about CBT for schizophrenia that have surfaced in the past year, one that it has more pronounced effects in treatment-resistant patients, ${ }^{4}$ and the other that it is most effective when delivered in an individually tailored, formulation-based form. ${ }^{5}$ Both these claims are based on meta-analyses that included only quite limited numbers of studies (12 and 13 respectively) and so could benefit from being examined in the full data-set of available studies.

1 Jauhar S, McKenna PJ, Radua J, Fung E, Salvador R, Laws KR. Cognitivebehavioural therapy for the symptoms of schizophrenia: systematic review and meta-analysis with examination of potential bias. Br J Psychiatry 2014; 204: 20-9.

2 National Institute for Health and Care Excellence. Psychosis and Schizophrenia in Adults: Treatment and Management (NICE Clinical Guideline CG178). NICE, 2014

3 Taylor M, Perera U. NICE CG178 Psychosis and Schizophrenia in Adults: Treatment and Management - an evidence-based guideline? Br J Psychiatry 2015; 206: 357-9.

4 Burns AM, Erickson DH, Brenner CA. Cognitive-behavioral therapy for medication-resistant psychosis: a meta-analytic review. Psychiatr Serv 2014; 65: 874-80.

5 van der Gaag M, Valmaggia LR, Smit F. The effects of individually tailored formulation-based cognitive behavioural therapy in auditory hallucinations and delusions: a meta-analysis. Schizophr Res 2014; 156: 30-7.

P. J. McKenna, MB ChB, FIDMAG Germanes Hospitalàries Research Foundation and CIBERSAM, Spain. Email: mckennapeter1@gmail.com; K. R. Laws, PhD, School of Life and Medical Sciences, University of Hertfordshire, UK; S. Jauhar, MB, ChB, BSC (Hons), Department of Psychosis Studies, Institute of Psychiatry, Psychology and Neuroscience, London, UK

doi: 10.1192/bjp.207.3.269a

\section{Should adherence to antidepressants be judged in isolation in ischaemic heart disease?}

We read the article by Krivoy et $a l^{1}{ }^{1}$ addressing an important clinical issue of medication adherence, especially antidepressants in patients with ischaemic heart disease, and its impact on mortality rates. The authors must be congratulated for evaluating the data of such a large sample after controlling for many known covariates. However, there are certain issues which require clarification, before accepting the 1:1 relationship of adherence to antidepressants only and reduced mortality rate.

First, it is unlikely that the patients would be adherent or nonadherent to antidepressants in isolation; hence, it is possible that those who were adherent to antidepressants were also adherent to other medications and this overall adherence to medications led to reduction in mortality rates. As a result, there is a need to provide the data in terms of adherence to other medications and include these as a covariate. Second, with such a large sample size, the authors should have evaluated the effect of each antidepressant or class of antidepressant on mortality. This is important from a clinical practice point of view, because this could have provided information about which antidepressants are more useful. Third, for assessing the confounding effect of comorbidity, the authors used the Charlson comorbidity index, which is considered to be a good predictive marker for mortality.
However, it is important to note that the index does not take dyslipidaemia into account. Accordingly, a covariate which is an important risk factor for mortality in patients with ischaemic heart disease could have been left out. Fourth, certain other covariates that can also influence mortality, for example alcohol use or dependence, were not taken into account. Fifth, although the authors have acknowledged that information on causes of death was not evaluated, it remains an important limitation. Sixth, the authors have not evaluated the prescribed doses in terms of being in the therapeutic range or not. This is important because antidepressants such as amitriptyline and duloxetine are prescribed by physicians at lower doses for indications other than depression or anxiety. Seventh, in the study, about three-quarters of patients were aged 65 years or older, with $38 \%$ of the study sample aged more than 74 years. If it is presumed that many of these patients were dependent on others for intake and purchasing of the medication, this should also be evaluated. Last, adherence to antidepressants was assessed in terms of medication possession ratio. In real terms this does not suggest that patients would have taken all the doses which they purchased. It is often a clinical experience that although patients purchase the prescribed medications, they do not take all the purchased medications. Consequently the authors would have overestimated the medication adherence.

1 Krivoy A, Balicer RD, Feldman B, Hoshen M, Zalsman G, Weizman A, et al. Adherence to antidepressant therapy and mortality rates in ischaemic heart disease: cohort study. Br J Psychiatry 2015; 206: 297-301.

Sandeep Grover, Associate Professor, Postgraduate Institute of Medical Education and Research, Chandigarh, India. Email: drsandeepg2002@yahoo.com; Mehdi Abbas, Postgraduate Institute of Medical Education and Research, Chandigarh, India

doi: 10.1192/bjp.207.3.270

Authors' reply: We thank Grover \& Abbas for their thoughtful comments on our paper. Most of the points they raise are appropriate and valid. Unfortunately, analysis of a large database (nationwide scale) has its strengths and limitations, including lack of access to some variables, as they suggested. The findings in our paper are indeed associational and not causative. Therefore, any notion regarding the causal effect of antidepressant adherence on mortality is speculative and validation in a prospective interventional study is required. It is possible that adherence to antidepressant treatment affects survival through moderators that were not examined in our study. Nevertheless, it appears that better adherence to antidepressants in patients with ischaemic heart disease is associated with increased survival rate. It is of note that our measure of adherence is unique in combining data regarding both prescribed and purchased prescriptions (unfortunately we did not have data on actual consumption of the pills). Most of the epidemiological studies on adherence use only purchase data as a measure of adherence. Therefore, we believe that our adherence measure reflects better the level of antidepressant adherence compared with previous similar studies.

Amir Krivoy, Clalit Research Institute, Tel Aviv, Geha Mental Health Center, Petah Tiqva, and Sackler Faculty of Medicine, Tel Aviv University, Tel Aviv, Israel. Email: amir.krivoy@gmail.com; Ran D. Balicer, Clalit Research Institute, Tel Aviv, Public lealth Depatment, Faculty of Health Sciences, Ben-Gurion University, Be iscitis Isael, Becca Feldman, Moshe Hoshen, Clalt Research instite, Tel Aviv, Is Gil Zalsman, Geha Mental Health Center, Petah Tiqva, Sackler Faculty of Medicine, Tel Aviv University, Tel Aviv, Israel, and Division of Molecular Imaging and Neuropathology, Department of Psychiatry, Columbia University, New York, USA; Abraham Weizman, Geha Mental Health Center, Petah Tiqva, Felsenstein Medical Research Center, Sackler Faculty of Medicine, Tel Aviv University, Tel Aviv: Gal Shoval, Clalit Research Institute, Tel Aviv, Geha Mental Health Center, Petah Tiqva, and Sackler Faculty of Medicine, Tel Aviv University, Tel Aviv, Israel.

doi: 10.1192/bjp.207.3.270a 